\title{
Spontaneous membrane formation and self-encapsulation of active rods in an inhomogeneous motility field
}

\section{Citation for published version (APA):}

Grauer, J., Löwen, H., \& Janssen, L. M. C. (2018). Spontaneous membrane formation and self-encapsulation of active rods in an inhomogeneous motility field. Physical Review E, 97(2), [022608].

https://doi.org/10.1103/PhysRevE.97.022608

DOI:

10.1103/PhysRevE.97.022608

Document status and date:

Published: 13/02/2018

\section{Document Version:}

Publisher's PDF, also known as Version of Record (includes final page, issue and volume numbers)

\section{Please check the document version of this publication:}

- A submitted manuscript is the version of the article upon submission and before peer-review. There can be important differences between the submitted version and the official published version of record. People interested in the research are advised to contact the author for the final version of the publication, or visit the $\mathrm{DOI}$ to the publisher's website.

- The final author version and the galley proof are versions of the publication after peer review.

- The final published version features the final layout of the paper including the volume, issue and page numbers.

Link to publication

\section{General rights}

Copyright and moral rights for the publications made accessible in the public portal are retained by the authors and/or other copyright owners and it is a condition of accessing publications that users recognise and abide by the legal requirements associated with these rights.

- Users may download and print one copy of any publication from the public portal for the purpose of private study or research.

- You may not further distribute the material or use it for any profit-making activity or commercial gain

- You may freely distribute the URL identifying the publication in the public portal.

If the publication is distributed under the terms of Article 25fa of the Dutch Copyright Act, indicated by the "Taverne" license above, please follow below link for the End User Agreement:

www.tue.nl/taverne

Take down policy

If you believe that this document breaches copyright please contact us at:

openaccess@tue.nl

providing details and we will investigate your claim. 


\title{
Spontaneous membrane formation and self-encapsulation of active rods in an inhomogeneous motility field
}

\author{
Jens Grauer, ${ }^{1}$ Hartmut Löwen, ${ }^{1}$ and Liesbeth M. C. Janssen ${ }^{1,2, *}$ \\ ${ }^{1}$ Institute for Theoretical Physics II: Soft Matter, Heinrich-Heine University Düsseldorf, Universitätsstraße 1, 40225 Düsseldorf, Germany \\ ${ }^{2}$ Theory of Polymers and Soft Matter, Department of Applied Physics, Eindhoven University of Technology, \\ P. O. Box 513, 5600MB Eindhoven, The Netherlands
}

(Received 10 July 2017; revised manuscript received 8 January 2018; published 13 February 2018)

\begin{abstract}
We study the collective dynamics of self-propelled rods in an inhomogeneous motility field. At the interface between two regions of constant but different motility, a smectic rod layer is spontaneously created through aligning interactions between the active rods, reminiscent of an artificial, semipermeable membrane. This "active membrane" engulfes rods which are locally trapped in low-motility regions and thereby further enhances the trapping efficiency by self-organization, an effect which we call "self-encapsulation." Our results are gained by computer simulations of self-propelled rod models confined on a two-dimensional planar or spherical surface with a stepwise constant motility field, but the phenomenon should be observable in any geometry with sufficiently large spatial inhomogeneity. We also discuss possibilities to verify our predictions of active-membrane formation in experiments of self-propelled colloidal rods and vibrated granular matter.
\end{abstract}

DOI: 10.1103/PhysRevE.97.022608

\section{INTRODUCTION}

Active materials are composed of autonomously moving agents that steadily consume energy while they are in motion. With only a small set of physical ingredients, they can mimic the complex behavior seen in living systems, such as swarming and flocking, directional motion, energy-fueled transport, clustering, and bacterial turbulence [1-4]. Over the past decade, numerous artificial active-matter systems have been designed and intensely studied, ranging from synthetic colloidal microswimmers on the micron scale to self-propelled vibrated granulates on the macroscopic scale. In many cases, the interactions between neighboring active particles are aligning such that they propel towards the same direction, giving rise to a flocking effect [5]. A relatively new avenue of research focuses on inhomogeneous motility fields, in which the particle self-propagation speed depends on the spatial coordinate. This is frequently encountered in actual biological or artificial systems where the swimming speed depends on an external stimulus, such as an externally imposed chemical [6-11], light $[12,13]$, or flow field $[14,15]$ of the solvent. Both linear gradients in motility $[6,13,16]$ and stepwise constant motility fields [17] have been studied but also more complicated motility ratchets $[13,18]$ and even motility waves propagating in time [19-22]. In general, in regions of low motility, active particles are dynamically trapped as they move much slower there, causing them to become locally pinned. The trapping efficiency has been recently studied in detail for nonaligning self-propelled spheres [17].

Here we explore the behavior of self-propelled rods with aligning interactions in inhomogeneous motility fields that are stepwise constant. We demonstrate that the combination

*L.M.C.Janssen@tue.nl of alignment and spatial inhomogeneity allows active matter to self-organize into nonequilibrium membranelike stuctures, reminiscent of colloidal [23,24] and biological lipid membranes $[25,26]$. In fact, at the interface where two regions of constant but different motility meet, a smectic-like rod layer is spontaneously created that acts as an effectively semipermeable "active membrane." In analogy to lipid membranes, this active self-organized structure leads to spontaneous compartmentalization and can be penetrated by other particles, and the number of particles forming the membrane is not fixed but fluctuating. The active membrane engulfes rods which are stuck in low-motility regions, an effect which we call self-encapsulation. Self-encapsulation can be understood as a self-organized "fence" around trapped rods which drastically enhances the trapping efficiency and thus naturally leads to compartmentalization of active particles [27]. It is worthwhile to mention that this self-organized trapping is qualitatively different from motility-induced phase separation, which occurs in homogeneous motility fields [28] and from capturing selfpropelled rods in wedgelike obstacles where the trapping is induced by geometry [29-31]. Moreover, the active membranes found here are different from active nematic films driven by anchoring and patterning [32-34].

Our results are gained by computer simulations of selfpropelled rod models [35-38] confined on a two-dimensional (2D) surface. We consider two geometries: a spherical surface in which the region of lower motility covers one hemisphere or a smaller surface area, and a 2D planar surface with periodic boundary conditions, in which one half of the surface is associated with a lower motility. We will focus mainly on the topology of the compact sphere, since it naturally gives rise to only a single interface and has also recently attracted interest due to its rich curvature- and topology-induced active-particle dynamics [39-46]. However, for the membrane-formation process reported here, the spherical topology is not a crucial 
ingredient: Indeed, we will show that self-encapsulation at the interface between two different motilities also occurs similarly for rod motion in the plane and that in fact the aligning interactions are the crucial factor. Moreover, we will discuss the similarity between the self-encapsulation process and the growth of smectic phases out of an isotropic phase that is impeded by rods lying perpendicular to the smectic layer [47], an effect known as "self-poisoning" in passive systems [48]. Our predictions may be verified in experiments of, e.g., self-propelled colloidal Janus rods steered by external light intensity [49,50], active microtubuli in a motility assay with varying kinesin motor concentrations [51], and vibrated granular matter [52]. Furthermore, our findings are of relevance for rodlike bacteria in different motility environments [53].

The paper is organized as follows. We first give an overview of the model systems used in the simulations, followed by a discussion of the structural and dynamical properties of the self-organized active membrane. We pay special attention to the encapsulation dynamics by probing time-correlation functions of the particle density on one side of the membrane. Last, we establish the robustness of the spontaneous membraneformation process by varying several parameters of the motility field. We close with concluding remarks and a perspective on possible experimental realizations of our system.

\section{SIMULATION MODEL}

Our simulation model describes self-propelled particles undergoing Brownian motion on a $2 \mathrm{D}$ surface with spacedependent motility. Explicitly, we have extended the models used by Janssen et al. [44] and Wensink et al. [37], which provide a minimal description for microswimmers confined to a spherical and 2D planar square surface with periodic boundary conditions, respectively, to the inhomogeneous case. In all simulations, the system is composed of $N$ rods of length $\ell$ that all experience a space-dependent self-propulsion force along their longitudinal rod axis $\hat{\mathbf{u}}_{i}$, where $i$ is the particle index [see Fig. 1(a)]. We choose the magnitude of the active force, $F\left(x_{i}\right)$, to be stepwise dependent on the Cartesian $x$ coordinate of the rods' center-of-mass positions $\mathbf{r}_{i} \equiv\left(x_{i}, y_{i}, z_{i}\right)$ [see Fig. 1(b)]:

$$
F\left(x_{i}\right)=\left\{\begin{array}{ll}
F_{1} & \text { if } x_{i} \leqslant 0 \\
F_{2} & \text { if } x_{i}>0
\end{array},\right.
$$

with $F_{1}$ and $F_{2}$ denoting constants. For both the spherical and planar confining surface, we place the origin of our coordinate system in the center, so that the low- and high-motility regions comprise equal surface areas. The special case $F_{1}=F_{2}$ reduces to the homogeneous scenarios of Refs. [37,44]. To account for steric repulsion among the particles, we represent each rod as a rigid chain of $m$ spherical segments and let all segment-segment pairs belonging to different rods interact through a repulsive Yukawa potential. The total interaction energy between two rods is given by

$$
U_{i j}=\frac{U_{0}}{m^{2}} \sum_{\alpha=1}^{m} \sum_{\beta=1}^{m} \frac{\exp \left(-r_{i j, \alpha \beta} / \lambda\right)}{r_{i j, \alpha \beta}},
$$

where $U_{0}$ is the potential amplitude, $r_{i j, \alpha \beta}$ is the Euclidean distance between segment $\alpha$ of $\operatorname{rod} i$ and segment $\beta$ of $\operatorname{rod} j$, (a)

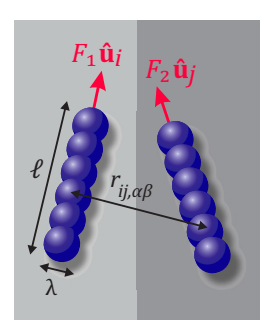

(b)

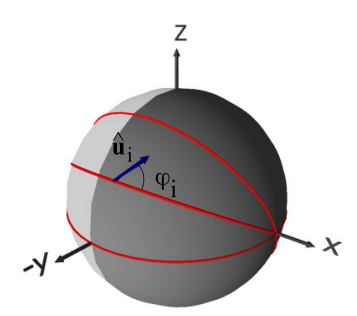

(c)

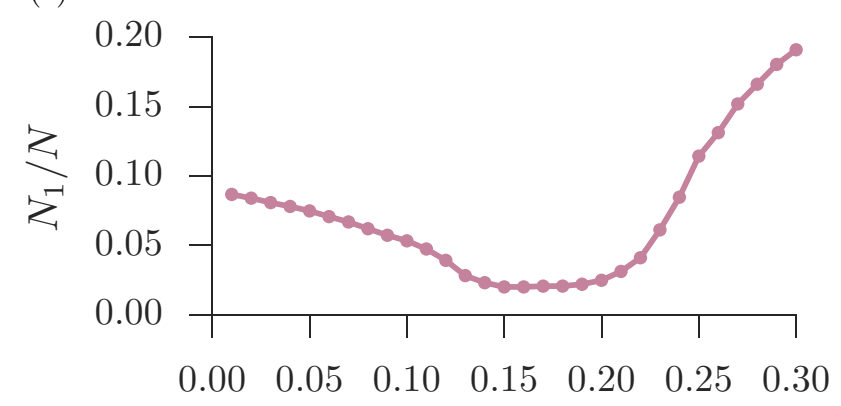

(d)

$\phi$

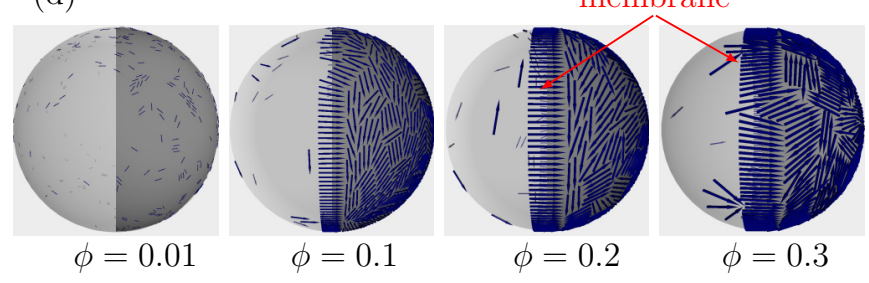

FIG. 1. (a) Sketch of the active-rod model. A light-gray background corresponds to a region with higher activity $F_{1}$, and dark-gray shading corresponds to a lower activity $F_{2}$. (b) Illustration of the coordinate system used for simulations on a spherical surface. The sphere is centered around the origin, and the region associated with the higher self-propulsion speed $F_{1}$ lies on the negative $x$ axis. The angle $\varphi_{i}$ represents the angle between a rod's orientation vector $\hat{\mathbf{u}}_{i}$ and the rotated meridian perpendicular to the boundary. (c) Average fraction of particles $N_{1} / N$ residing on the "fast" $x \leqslant 0$ hemisphere as a function of packing fraction $\phi$ for a system of $N=200$ rods with particle aspect ratio $a=10$ and activity ratio $F_{1} / F_{2}=10$. (d) Representative snapshots of steady-state configurations at various packing fractions, obtained after a total simulation time of $60000 \tau$.

and $\lambda$ is the screening distance that also serves as the unit of length [see Fig. 1(a)]. For the spherical topology, we follow Ref. [44] and constrain the rods such that $\mathbf{r}_{i}$ always lies on the sphere and $\hat{\mathbf{u}}_{i}$ lies in the plane tangent to the sphere at position $\mathbf{r}_{i}$. Within such a local tangent plane, the dynamics can be treated as effectively two dimensional, and hence we simulate all dynamics by integrating the 2D overdamped Langevin (Brownian) equations of motion,

$$
\begin{aligned}
\dot{\mathbf{r}}_{i} & =\boldsymbol{\mu}_{T}\left[-\nabla_{\mathbf{r}_{i}} U+F\left(x_{i}\right) \hat{\mathbf{u}}_{i}\right], \\
\dot{\hat{\mathbf{u}}}_{i} & =-\boldsymbol{\mu}_{R} \nabla_{\hat{\mathbf{u}}_{i}} U,
\end{aligned}
$$

where the dots denote time derivatives, $U=\frac{1}{2} \sum_{i, j \neq i} U_{i j}$, and $\nabla_{\hat{\mathbf{u}}_{i}}$ is the gradient on the unit circle. The matrices $\boldsymbol{\mu}_{T}$ and $\boldsymbol{\mu}_{R}$ represent inverse translational and rotational friction tensors, 
respectively, which are defined as

$$
\begin{gathered}
\boldsymbol{\mu}_{T}=\mu_{0}\left[\mu_{\|} \hat{\mathbf{u}}_{i} \otimes \hat{\mathbf{u}}_{i}+\mu_{\perp}\left(\mathbf{I}-\hat{\mathbf{u}}_{i} \otimes \hat{\mathbf{u}}_{i}\right)\right], \\
\boldsymbol{\mu}_{R}=\mu_{0} \mu_{R} \mathbf{I} .
\end{gathered}
$$

Here $\mu_{0}$ is a mobility coefficient, $\mathbf{I}$ is the $2 \times 2$ unit matrix, and $\otimes$ stands for the dyadic product, and for the parameters $\mu_{\|}, \mu_{\perp}$, and $\mu_{R}$ we use the standard expressions for rodlike macromolecules as given in Ref. [54]. We adopt characteristic units such that $\lambda=1, \mu_{0}=1$, and for the unit of activity we set $F_{0}=1$, so that time is measured in units of $\tau=$ $\lambda /\left(\mu_{0} F_{0}\right)$. For the potential we take $U_{0}=250$ and a cutoff distance of $r_{c}=6 \lambda$, and the number of segments per rod is chosen as $m=\lceil 14 a / 8\rceil$, where $a=\ell / \lambda$ is the rod aspect ratio. Equation (3) is propagated using a Euler integration scheme with a discrete time step of $0.01 \tau$. For simplicity we have ignored any stochastic noise and hydrodynamic interactions (HI) - implying that the dynamics is governed solely by the repulsive pair interactions and self-propulsion forces - but we have verified that the membrane is also stable against small thermal noise, as will be shown below. Due to the neglect of HI, our model is particularly suitable for dry active matter, but we note that HI-free simulations can also accurately reproduce the complex behavior seen in hydrodynamic models, including active turbulence [37] and compartmentalization of active spinners [27].

\section{MEMBRANE FORMATION AND STRUCTURE}

We first explore the emergent structural and dynamical properties as a function of the packing fraction $\phi$, defined for the spherical surface as $\phi=N \ell \lambda /\left(4 \pi R^{2}\right)$, where $R$ is the radius of the confining sphere, and for the planar surface as $\phi=N \ell \lambda / \ell_{\text {box }}^{2}$, where $\ell_{\text {box }}$ is the width of the square simulation box. Let us first focus on the spherical-surface case. Figure 1(c) shows the fraction of rods on the high-motility $x \leqslant 0$ hemisphere, $N_{1} / N$, as a function of $\phi$ for a system of $N=200$ rods with aspect ratio $a=10$ and self-propulsion strengths $F_{1}=1 F_{0}$ and $F_{2}=0.1 F_{0}$. In the dilute limit of $\phi \rightarrow 0$, all rods behave as free particles that spend a fraction $F_{2} /\left(F_{1}+F_{2}\right)$ of the time on the left hemisphere, implying $N_{1} / N=0.09$, which indeed we observe numerically. As the density increases up to $\phi \approx 0.2$, however, we find a remarkable effect: The "fast" region with $x \leqslant 0$ becomes depleted and an excessive amount of particles will reside at the hemisphere with the lower self-propulsion speed $F_{2}$. The reason for this becomes evident from the particle snapshots, Fig. 1(d): At the boundary between the two hemispheres, particles self-organize into a membranelike structure that effectively prevents particles from leaving the "slow" $x>0$ region, thus acting as a self-encapsulation mechanism. The formation of this membrane arises from three crucial ingredients: (i) the spatial inhomogeneity of the motility, $F_{1}>F_{2}$, which naturally imposes an inhomogeneous density profile; (ii) a sufficiently high packing fraction, which allows for saturation of rods on the $x>0$ hemisphere; and (iii) aligning interactions, which emerge from pair collisions between the active rods. Indeed, we have verified that the membrane structure disappears if $F_{1} \approx F_{2}, \phi \ll 0.2$, or $a \ll 10$. We also note that the formation of the membrane is fostered by the periodicity of the sphere: If a rod is able to permeate through the interface and move into the $x \leqslant 0$ region, then it will swim across the entire hemisphere and eventually collide with membrane-forming particles on the other side, consequently causing it to align and becoming part of the membrane itself. Through a similar mechanism, we see that for higher packing fractions $\phi>0.2$, where the "slow" $x>0$ hemisphere is fully saturated with particles, "hedgehog" structures [36] appear on the "fast" $x \leqslant 0$ side of the membrane. Thus, a polar ordering of particles oriented toward the domain associated with lower motility emerges naturally near the interface.

For rods residing on a flat $2 \mathrm{D}$ surface with periodic boundary conditions, the observed behavior is very similar to that on the sphere: At packing fractions $\phi \approx 0.2$ a clear membrane structure appears at the interface between the regions of different motilities. Due to the periodic boundary conditions, we now find two separate membranes at $x=0$ and $|x|=\ell_{\text {box }} / 2$ which encapsulate the rods in the low-motility region from opposing sides. Figure 2(a) shows a typical snapshot of this scenario for $N=800$ rods with aspect ratio $a=16$ and $F_{1} / F_{2}=$ 10. As in the spherical case, a membrane is formed only when the rods are sufficiently elongated to induce sufficiently strong aligning pair collisions, and indeed the membrane structure becomes increasingly distorted as the rod aspect ratio decreases.

To unambiguously confirm that aligning interactions are crucial, we have also performed 2D calculations for spherical particles with $a=1$ that experience no torque during collision. In this case, particle reorientation may only occur through rotational Brownian diffusion. In order to account for such diffusional motion and thus to allow for a fair comparison between the dynamics of rods and spheres, we have extended our simulation model to finite Péclet number Pe $=\mu_{0} F_{1} / \sqrt{D_{\|} D_{R}}$, where $D_{\|}=\mu_{0} \mu_{\|} k_{B} T$ and $D_{R}=\mu_{0} \mu_{R} k_{B} T$ are translational and rotational diffusion coefficients, respectively, $k_{B}$ is the Boltzmann constant, and $T$ is a temperature. For elongated rods, we have verified that the membrane structure is robust against thermal Brownian translational and rotational noise. For spherical particles, however, we find a markedly different pattern: In the absence of explicitly aligning interactions, the particles form an active crystalline phase at $\phi=0.2$ that covers the entire surface homogeneously. Figure 2(b) shows a snapshot of such a phase for $N=800, a=1, \mu_{\|}=\mu_{R}=$ $1, \mathrm{Pe}=100$, and $F_{1} / F_{2}=10$. Here the average number of particles is the same in the $x<0$ and $x>0$ regions, and, consequently, the lattice constants are identical for the highand low-motility domains. We have checked that the observed crystalline pattern for this packing fraction also appears for lower Pe values and higher particle numbers (up to $N=4000$ ), thus ruling out possible finite-size effects. As the packing fraction decreases to $\phi=0.1$, however, the high-motility region becomes fluidized, as shown in Fig. 2(c). Thus, rather than forming a membrane, the motility edge for spherical particles becomes a fluid-crystal interface, with the fluid on the high-activity and the hexagonal crystal on the low-activity side. This reveals that, at the appropriate density range, one can steer fluid and crystal phases at wish by inhomogeneous motility fields. We note that the formation of a hexagonally ordered phase of active Brownian particles in a low-motility region has also been reported by Magiera and Brendel [17] for 
(a)

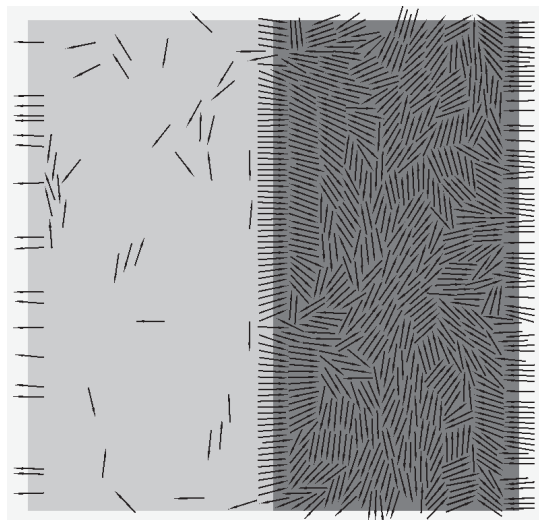

(b)

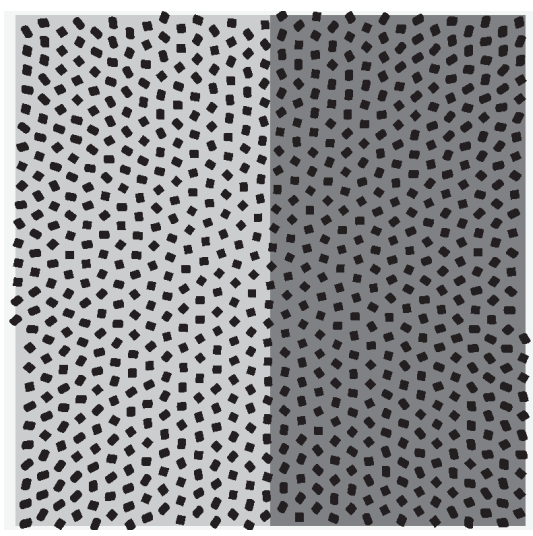

(c)

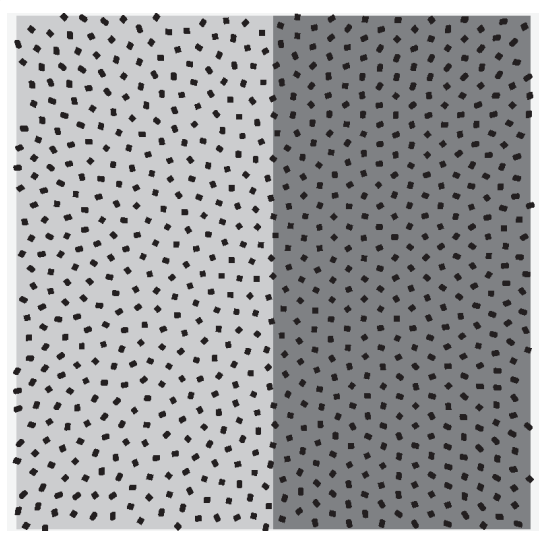

FIG. 2. Representative snapshots of steady-state configurations for $N=800$ particles and activity ratio $F_{1} / F_{2}=10$ on a $2 \mathrm{D}$ surface with periodic boundary conditions. (a) Particle aspect ratio $a=16$ and packing fraction $\phi=0.2$; (b) particle aspect ratio $a=1$ and packing fraction $\phi=0.2$, with $\mu_{\|}=\mu_{R}=1$ and $\mathrm{Pe}=100$; and (c) particle aspect ratio $a=1$ and packing fraction $\phi=0.1$, with $\mu_{\|}=\mu_{R}=1$ and $\mathrm{Pe}=100$. In the latter two cases, the particles are spherically shaped and experience no aligning torques during collision.

spherical particles with a shorter-ranged repulsive interaction potential. The detailed Brownian dynamics of spherical active particles in inhomogeneous motility fields will be discussed in a separate publication; for the present work, we only emphasize that aligning torques are necessary to form the here-reported membrane structure.
In order to characterize the structure of the self-organized membrane of active rods, we calculate the density profile $\rho(x)$, the polar order parameter $P(x)$, and the nematic order parameter $S(x)$, which are defined as

$$
\begin{gathered}
\rho(x)=\sum_{i=1}^{N}\left\langle\delta\left(x-x_{i}\right)\right\rangle, \\
P(x)=\frac{1}{N} \sum_{i=1}^{N}\left\langle\cos \left(\varphi_{i}\right)\right\rangle_{x}, \\
S(x)=\frac{1}{N} \sum_{i=1}^{N}\left\langle 2 \cos ^{2}\left(\varphi_{i}\right)-1\right\rangle_{x},
\end{gathered}
$$

where $\langle\cdot\rangle$ denotes an ensemble average and $\langle\cdot\rangle_{x}$ an average under the constraint that the position of the $i$ th $\operatorname{rod}$ is at $x_{i}=x$. For the spherical confining surface, $\varphi_{i}$ is the angle between the $\operatorname{rod}$ axis $\hat{\mathbf{u}}_{i}$ and rotated meridian perpendicular to the boundary [see Fig. 1(b)], while for the 2D plane $\varphi_{i}$ represents the angle between $\hat{\mathbf{u}}_{i}$ and the positive $x$ axis. In both cases, the values of $P(x)$ and $S(x)$ can range from +1 to -1 . Figure 3 depicts the $\rho(x), P(x)$, and $S(x)$ profiles for systems with a fully developed membrane on a spherical and planar 2D surface, respectively, all calculated for $N=800, \phi=0.2$, and $F_{1} / F_{2}=10$. The data are averaged over 30 and 50 independent trajectories, respectively.

The average density profiles, Figs. 3(a) and 3(d), indicate that almost all particles reside on the region with low motility, $x>0$, confirming a high trapping efficiency on both the spherical and planar surface. Moreover, in both cases, the membrane at $x \approx 0$ is composed of a large number of particles with nearly perfect parallel alignment along the $x$ axis, since $P(0) \approx 1$ [Figs. 3(b) and 3(e)] and $S(0) \approx 1$ [Figs. 3(c) and 3(f)]. Note that by symmetry, the membrane at $|x|=\ell_{\text {box }} / 2$ on the planar surface has the opposite polarization. Furthermore, in the 2D planar case, a second, third, and even fourth row of parallel-oriented particles is clearly visible at $x / \ell \simeq 1,2$, and 3 , respectively. For the spherical surface, however, this smectic ordering is frustrated by the curvature of the sphere, and only one additional layer of particles is apparent at $x / \ell \simeq 1$. We have verified that the smectic layering on the sphere is further enhanced when the inhomogeneity ratio increases, $F_{1} \gg F_{2}$.

Curiously, on both sides of the membrane, at $x / \ell \approx-0.8$ and $x / \ell \approx 0.8$, we find a subset of particles aligned perpendicular to the membrane-forming rods, as evidenced by the locally strongly negative nematic order parameter $S(x)$. Note that this occurs similarly for the spherical and planar case. The origin of the transverse order in the high-density (i.e., low-motility) region is, however, qualitatively different from that occuring in the low-density region. In the high-density region, intralayer particles with perpendicular ordering arise from packing: This effect occurs already in bulk equilibrium $[47,55]$ and was also found as a "self-poisoning" scenario for crystallizing hard-rod liquids by Schilling and Frenkel [48]. The perpendicularly oriented particles effectively hamper permeation of rods across the membrane, thus partially shielding the self-organized structure. On the low-density side, perpendicular ordering has mainly a dynamical origin. Particles which are perpendicular just repeatedly move around more times contributing therefore 

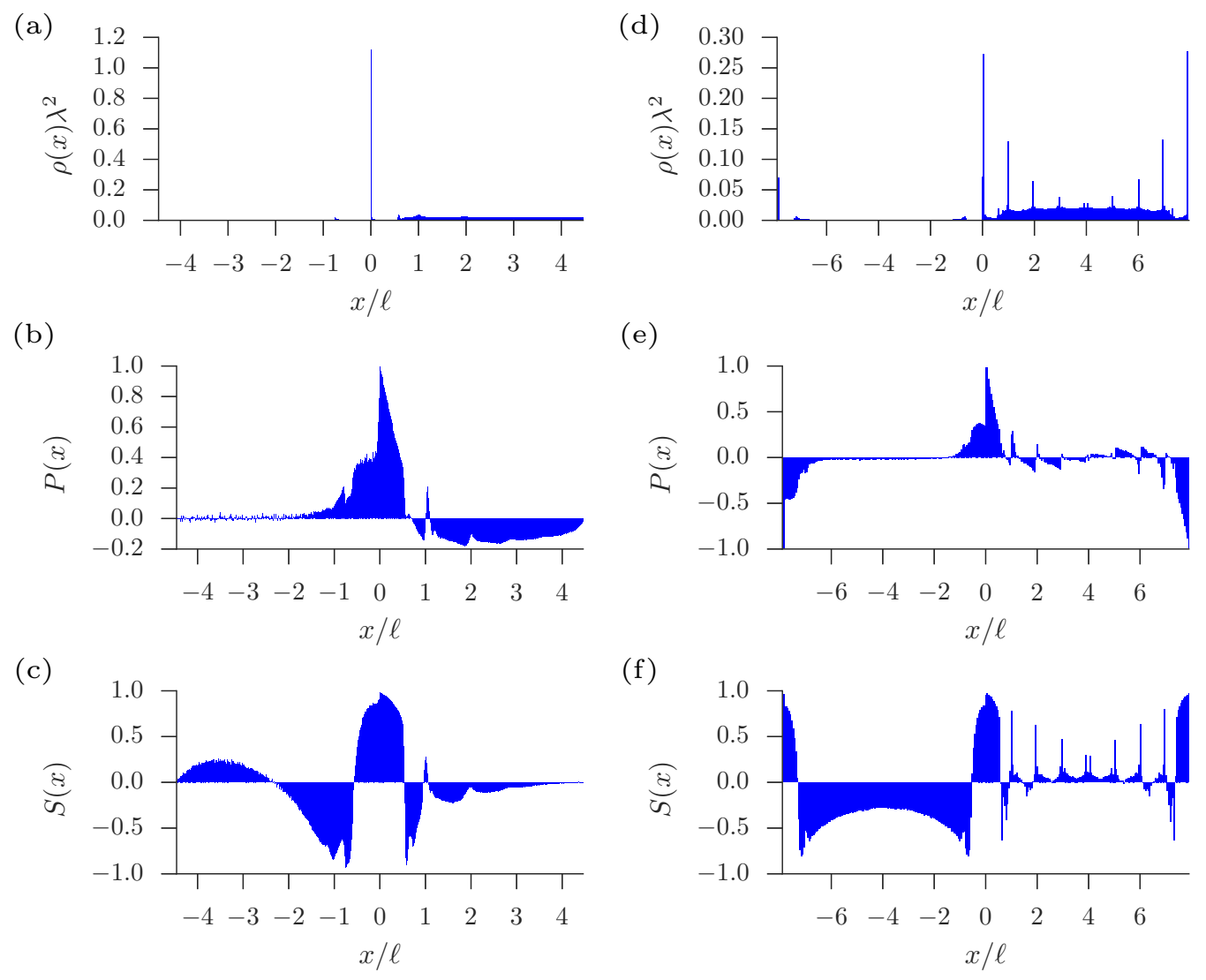

FIG. 3. Structural order parameters for $N=800$ active rods with aspect ratio $a=16$ at packing fraction $\phi=0.2$ and activity ratio $F_{1} / F_{2}=$ 10 , calculated for [(a)-(c)] a spherical confining surface, and [(d)-(f)] a 2D surface with periodic boundary conditions. [(a) and (d)] Average density profile $\rho(x) \lambda^{2}$, [(b) and (e)] polar order parameter $P(x)$, and [(c) and (f)] nematic order parameter $S(x)$. In all panels, the $x$ coordinate is normalized by the rod length $\ell=16 \lambda$.

more strongly to the average (both in the circular and planar situation). As the distance from the interface increases, the structural ordering becomes less distinct. For the spherical surface, this means that at the poles with $|x|=R$, the order parameters $S(x)$ and $P(x)$ both level off to zero due to symmetry. It may be seen in Fig. 3(c) that, when approaching the $x=-R$ pole of the low-density region, the nematic order parameter $S$ changes again sign at $x / \ell \simeq-2.3$. This is due to the definition of the latitudinal reference orientation, as a typical trajectory nearby the pole will be on average more likely to be parallel than perpendicular to the latitudinal direction. For the 2D planar case, the rods moving in the high-motility region $(x<0)$ have a weak propensity to remain perpendicular to the membrane-forming rods, leading to a net negative value of the nematic order parameter even in the middle between the two opposing membranes, at $x=-\ell_{\text {box }} / 4$; this is attributed to the finite size of the simulation box, which we have verified by comparing simulations for $N=800$ and $N=2000$. The net polarization is negligible; however, $P\left(-\ell_{\text {box }} / 4\right) \approx 0$, which naturally follows from the absence of any symmetry-breaking mechanism along the $y$ axis. Overall, we can conclude that the structure and encapsulation function of the membrane, which forms at the interface between a high- and low-motility region, is qualitatively similar for a spherical and planar surface, but the details of the particle ordering away from the membrane depend on the geometry and symmetry of the imposed confinement.

\section{PERMEATION DYNAMICS}

We next turn our attention to the permeation dynamics of the membrane and monitor how the concentration of particles in the high-motility region $(x \leqslant 0)$ evolves as a function of time. For simplicity we focus here on the spherical-surface case, since it gives rise to only a single membrane, but all results can be assumed to be qualitatively similar to the $2 \mathrm{D}$ planar case. We define an incoherent time-correlation function probing the single-particle dynamics,

$$
C_{s}(t)=\frac{1}{N} \sum_{i=1}^{N} \frac{\left\langle n_{i}(0) n_{i}(t)\right\rangle}{\left\langle n_{i}^{2}\right\rangle},
$$

where $n_{i}$ measures on which hemisphere a particle resides,

$$
n_{i}= \begin{cases}1 & \text { if } x_{i} \leqslant 0 \\ 0 & \text { if } x_{i}>0\end{cases}
$$

and a coherent time-correlation function probing the collective dynamics,

$$
C_{c}(t)=\frac{\left\langle N_{1}(0) N_{1}(t)\right\rangle}{\left\langle N_{1}^{2}\right\rangle},
$$

where $N_{1}=\sum_{i=1}^{N} n_{i}$. Furthermore, we also consider the average particle flux per unit time and unit length, $J /(\tau \lambda)$, which measures the net number of particles crossing the interface towards the high-motility region. In all cases, the average 
is taken over a maximum of 700 independent trajectories with a total simulation time up to $1.2 \times 10^{6} \tau$ each. In order to expedite the statistical averaging process, we here focus on smaller systems with $N=200$, but we have verified that the qualitative picture applies also for larger system sizes of $\mathcal{O}(1000)$.

As a reference case, let us first consider the dynamics in the dilute limit where particles behave as free swimmers and no membrane structure is formed. In this scenario, every rod will swim independently across a great circle of the sphere, spending time periods of relative duration $F_{2} /\left(F_{1}+\right.$ $\left.F_{2}\right)$ and $F_{1} /\left(F_{1}+F_{2}\right)$ on the $x \leqslant 0$ and $x>0$ hemispheres, respectively. The corresponding canonically averaged timecorrelation function $C_{s}(t)$ will, after a brief initial decay, oscillate around the average value $\left\langle n_{i}^{2}\right\rangle \equiv\left\langle n_{i}\right\rangle=F_{2} /\left(F_{1}+\right.$ $\left.F_{2}\right) \approx 0.09$ with a period of $\tau_{c}$, i.e., the time it takes a free particle to cover one great circle. Our simulations at $\phi=0.01$ numerically confirm this picture, as can be seen from Fig. 4(a). While in this case all particles may cross the interface at $x=0$ without experiencing any steric hindrance, the normalized particle flux $J /(\tau \lambda)$ is still close to zero, as shown in Fig. 4(e). This is simply due to the very low particle density and correspondingly large interface length, resulting in an almost negligible flux per unit of length.

At a slightly higher packing fraction of $\phi=0.05$, we witness the onset of membrane formation: particle collisions promote the formation of polar domains and rods accumulate on the region with lower activity. While not forming a fully developed membrane across the entire sphere, but rather a dynamic polar structure that is constantly dissolved and rebuilt locally, the membrane-like domains do transiently trap particles and effectively delay the crossing time between the two hemispheres. Indeed, the long-time value $\left\langle n_{i}\right\rangle$ is slightly lower than in the free-particle case, and, importantly, the oscillation period of $C_{s}(t)$ at $\phi=0.05$ is a factor of 1.5 larger than the time $\tau_{c}$ expected for noninteracting particles. To unambiguously establish that this is not merely a result of the increased particle density, we also compare our results against the homogeneous case with uniform activity $F_{1}=F_{2}=\frac{2}{11} F_{0}$, in which case the characteristic time $\tau_{c}$ is identical but the membrane is absent. Figure 4(b) reveals that this scenario would result in a $C_{s}(t)$ oscillation period of approximately 1.2 times $\tau_{c}$, thus confirming that the presence of the membrane delays the dynamics and gives rise to enhanced trapping and self-encapsulation. This trend continues as the packing fraction further increases to $\phi=0.1$, in which case the homogeneous reference scenario reveals oscillations in $C_{s}(t)$ with a period of $1.5 \tau_{c}$, while the inhomogeneous $C_{s}(t)$ data show only a single oscillation and decay to a lower long-time value, indicative of the fact that particles become more strongly trapped behind the membrane on the $x>0$ hemisphere. Figure 4(e) also shows that the net flux slightly decreases at $\phi=0.1$, consistent with an enhanced trapping effect due to the partially permeable membrane.

Finally, at a packing fraction of $\phi=0.15$, a "perfect" membrane is formed that covers the entire interface at $x=0$, and almost all remaining particles are encapsulated in the densely packed $x>0$ region. Here the membrane has completely lost its permeability and instead acts as a self-organized trapping barrier that fosters a maximal accumulation of rods on one side (a)

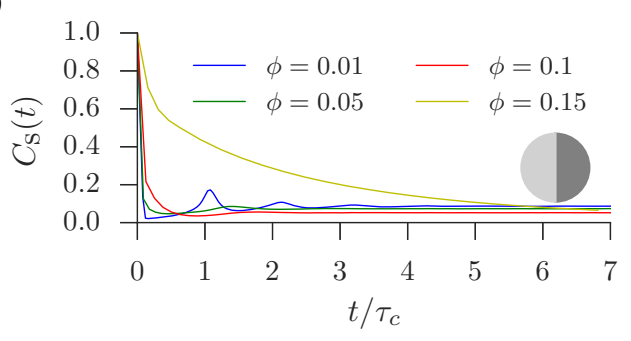

(b)

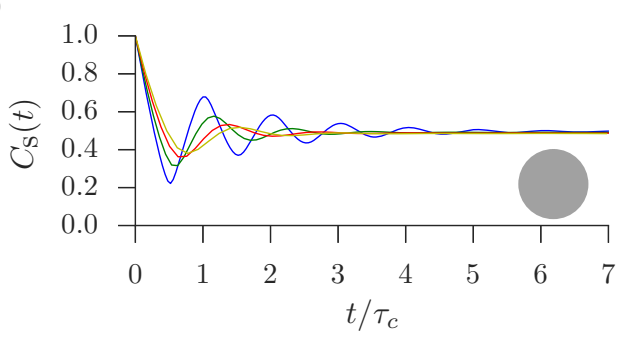

(c)

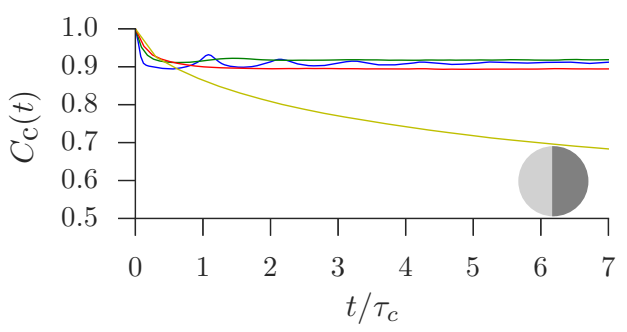

(d)

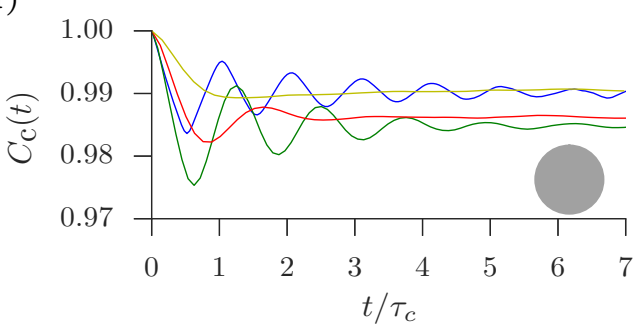

(e)

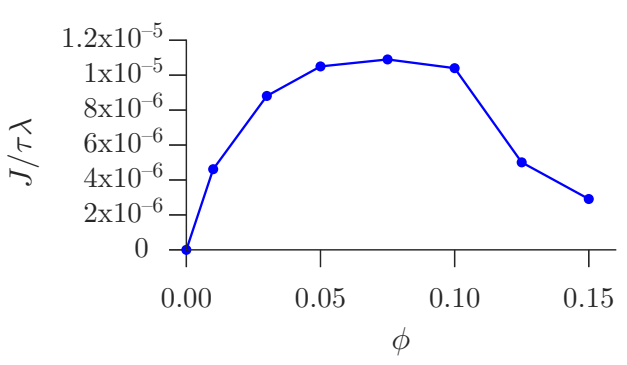

FIG. 4. [(a)-(d)] Time-correlation functions calculated for $N=$ 200 rods with $a=10$ at different packing fractions $\phi$. The top two panels show the incoherent $C_{s}(t)$ functions [Eq. (9)] for (a) inhomogeneous activity ratio $F_{1} / F_{2}=10$ and (b) homogeneous activity, i.e., $F_{1} / F_{2}=1$. Insets illustrate the motility field. The lower panels show the corresponding collective $C_{c}(t)$ functions [Eq. (11)] for (c) $F_{1} / F_{2}=10$ and (d) $F_{1} / F_{2}=1$. Time is given in units of the characteristic time $\tau_{c}$ in which a free particle will swim across one great circle of the sphere. All legends are as in panel (a). Panel (e) shows the average particle flux $J /(\tau \lambda)$ across the interface for $F_{1} / F_{2}=10$ as a function of packing fraction $\phi$.

of the sphere. A comparison of the time-correlation functions in Figs. 4(a) and 4(b) for $\phi=0.15$ confirms this picture: in the inhomogeneous $\left(F_{1} / F_{2}=10\right)$ case, there is not a single 
oscillation in $C_{s}(t)$ visible on the time scale considered in this work, and instead we observe only a very slow decay pattern in which a limited number of particles manages to change hemispheres. Conversely, the homogeneous $\left(F_{1} / F_{2}=1\right)$ case exhibits an oscillatory pattern with a period of $1.8 \tau_{c}$, implying that here particles can depart and reenter the "slow" $x>0$ hemisphere far more easily. From Fig. 4(c) we also see that the coherent time-correlation function $C_{c}(t)$ at $\phi=0.15$ decays to a much lower long-time value than at lower packing fractions, indicating that the particle number $N_{1}$ exhibits far greater fluctuations. This is due to the fact that $N_{1}$ is minimal when the encapsulation effect is maximal [cf. Fig. 1(a)], implying that any particle crossing the interface at $\phi=0.15$ will constitute a relatively large change in $N_{1}$ and thus a relatively strong decorrelation in $C_{c}(t)$. We also note that for all packing fractions considered here the permeation dynamics is governed predominantly by single-particle crossing events, rather than groups of collectively permeating particles, and indeed the decay of $C_{c}(t)$ is enslaved by $C_{s}(t)$.

As a final confirmation of the strong trapping effect at $\phi=0.15$, we observe a distinct drop in the particle flux at this packing fraction [Fig. 4(e)]. Collectively, these results thus unambiguously show that the self-organized membrane structure leads to high trapping efficiency and slow permeation dynamics, resulting in the spontaneous compartmentalization of particles. We have verified for Péclet numbers of $\mathrm{Pe}=$ 100 and 300 that the inclusion of thermal translational and rotational Brownian noise does not alter this qualitative picture but does lead to a higher effective permeability.

\section{DEPENDENCE ON GEOMETRIC PARAMETERS}

Let us finally investigate how robust the spontaneous membrane-formation process is against variations in the geometric parameters of the motility field, namely the surface area of the region associated with lower activity and the inhomogeneity ratio $F_{1} / F_{2}$. To vary the surface area we consider the case

$$
F\left(x_{i}\right)=\left\{\begin{array}{ll}
F_{1} & \text { if } x_{i} \leqslant x_{0} \\
F_{2} & \text { if } x_{i}>x_{0}
\end{array},\right.
$$

with $x_{0}>0$, so that the region of lower self-propulsion speed will become increasingly small as $x_{0}$ increases. Figure 5 shows representative shapshots for a system of $N=800$ rods on a spherical surface with $\phi=0.2$ and $F_{1} / F_{2}=10$, for different interface locations $x_{0}=0.55 R, 0.78 R$, and $0.97 R$. We find that up to $x_{0} \approx 0.9 R$, a membranelike smectic ordering is consistently formed across the interface but becomes more distorted as the low-motility region becomes smaller. For the packing fraction considered here, the surface area available in the low-motility domain is insufficient to accommodate all particles, even for small values of $x_{0}>0$. Hence, particles must inevitably reside in the region with higher activity, forming distorted smectic layers and hedgehog-like structures around the $F_{2}$ domain-similarly to what we found for the $x_{0}=0$ case at higher packing fractions. We thus conclude that the low-motility region acts as a "nucleation" core around which particles accumulate, even as this domain becomes completely saturated with particles. This phenomenon is akin to the behavior reported earlier for nonaligning active spheres in
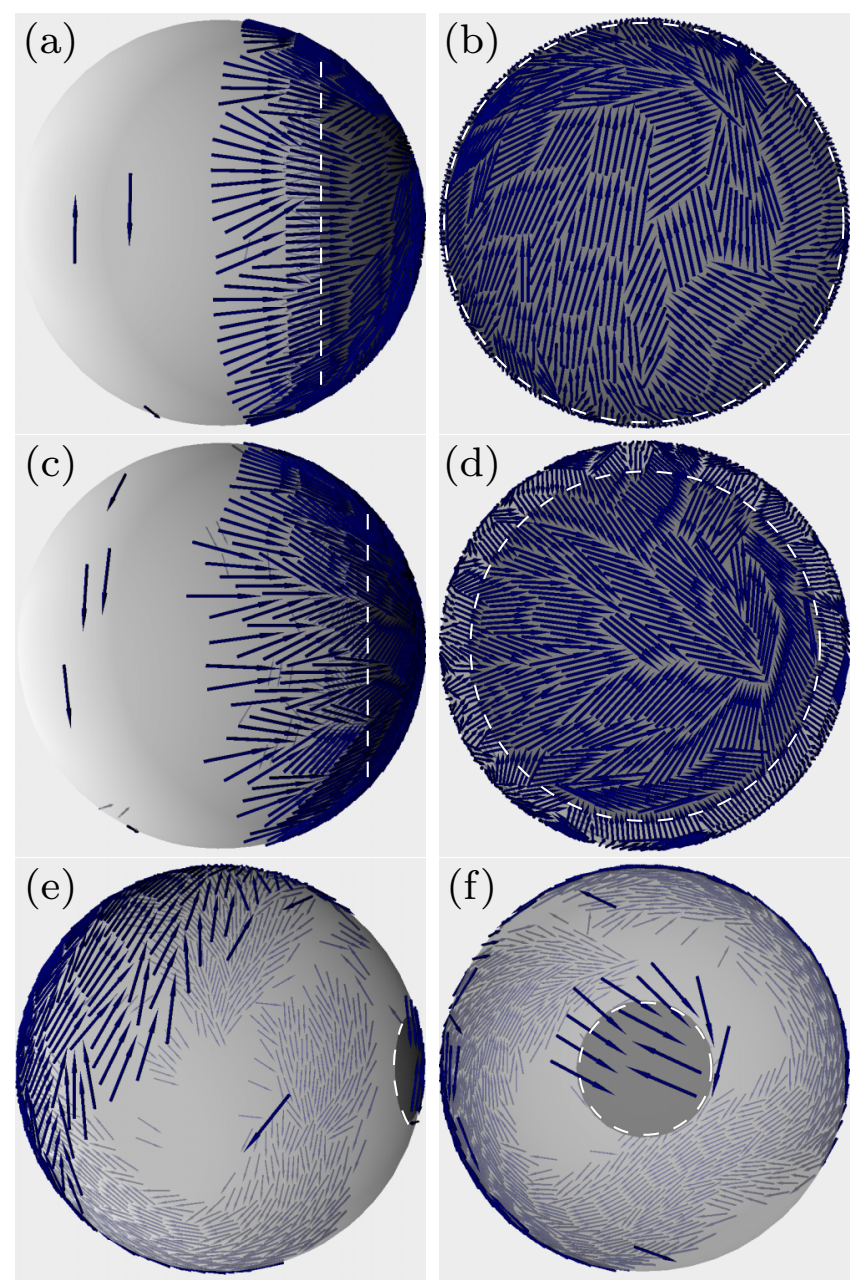

FIG. 5. Representative snapshots for a system of $N=800$ rods with aspect ratio $a=16$ and packing fraction $\phi=0.2$, with step locations of the motility field located at [(a) and (b)] $x_{0}=0.55 R$, $\left[(\mathrm{c})\right.$ and (d)] $x_{0}=0.78 R$, and [(e) and (f)] $x_{0}=0.97 R$, as indicated by the white dashed lines. Left panels show the $(x, z)$ plane and right panels the $(y, z)$ plane.

inhomogeneous media [17] and implies that even a small inhomogeneity can effectively trap an excessive number of particles. Consequently, this behavior may readily be exploited in applications that require spatial control over active particles. In the limit of $x_{0} \rightarrow R$, i.e., a vanishing low-motility region, we recover the homogeneous scenario of Ref. [44] which, for the rod aspect ratios and densities considered here, gives rise to a flocking state [cf. Figs. 5(e) and 5(f)].

Last, by varying the ratio between self-propulsion speeds, $F_{1} / F_{2}$, we confirm that the membrane structure is fostered by a strong inhomogeneity. Figure 6 shows the dimensionless density profiles $\rho(x) \lambda^{2}$ for a spherical surface with $N=800$, $\phi=0.2$, and $x_{0}=0$ with activity ratios $F_{1} / F_{2}=10,2$, and 1.1. It is clear that a larger difference in motilities leads to a more prominent membrane structure at the interface. As the values of $F_{1}$ and $F_{2}$ approach each other, the rods experience a smaller difference in self-propulsion speed and the density profile becomes more homogeneous across the entire sphere. In the limit of $F_{1}=F_{2}$ we again recover the flocking state of Ref. [44] for the spherical surface and of Ref. [37] for 

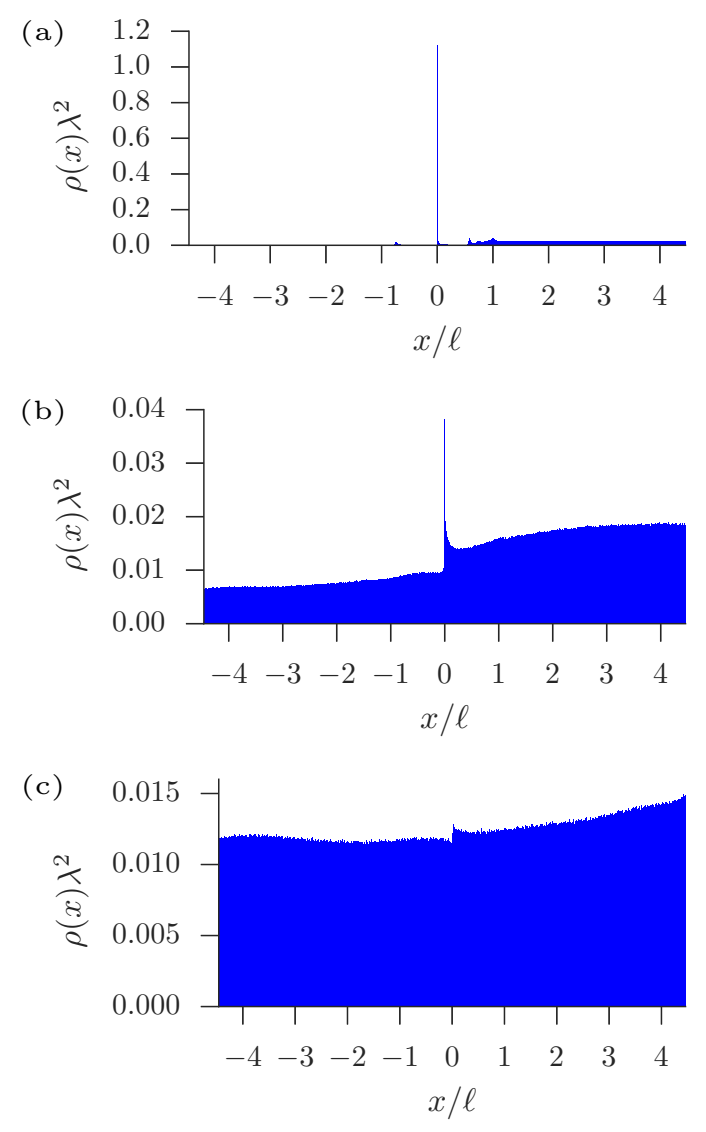

FIG. 6. Density profiles $\rho(x) \lambda^{2}$ for a system of $N=800$ rods with aspect ratio $a=16$, packing fraction $\phi=0.2$, and interface location $x_{0}=0$, for different activity ratios $F_{1} / F_{2}$ of (a) 10 , (b) 2 , and (c) 1.1 .

the planar 2D surface. This unambiguously confirms that spatial inhomogeneity is a crucial ingredient for the membrane formation and self-encapsulation of active rods.

\section{CONCLUSIONS}

In conclusion, we have established a link between the physics of membranes and self-propelled particles: In an inhomogeneous motility field, an active membrane is spontaneously formed by a competition between self-propulsion and rod interactions. The effect is robust and occurs in any geometry provided there is a steep jump in motility over the rod length. The active membrane encapsulates particles trapped in a low-motility region and significantly enhances the trapping efficiency. This possesses applications to capture and steer microswimmers efficiently via motility fields.

In principle it is possible to verify our predictions in experiments. One feasible realization consists of colloidal Janus rods driven by light $[49,50]$, which can be exposed to almost arbitrary motility landscapes [13]. Similar possibilities exist for self-propelled droplets [11] or modular microswimmers steered by an electrolyte gradient [56]. Moreover, rodlike bacteria at high concentrations $[37,53]$ may serve as another model system to observe smectic ordering in motility landscapes. Last, macroscopic rodlike granulates can be made active by vertical vibration [52,57] and different motilities can in principle be controlled by imposing an upper frictional zone parallel to the vibrating table. This kind of "dry" active matter is in particular an excellent realization of our model as hydrodynamic interactions are absent. For the future it would be interesting to also incorporate solvent-mediated hydrodynamic interactions between the rods into our model. Due to the constrained motion of the rods on the spherical surface this will be a highly nontrivial task.

Finally, there is an increasing interest in microscopic statistical theories for interacting active particles. Dynamical density functional theory [36,58-60] is an appropriate approach to obtain predictions for layering of rodlike particles. In order to describe the effects theoretically, these theories need to be supplemented and generalized towards an inhomogeneous motility field, see Ref. [22] for a recent study in this direction.

\section{ACKNOWLEDGMENTS}

H.L. acknowledges the DFG for support through project LO 418/20-1. L.C.M.J. thanks the Alexander von Humboldt Foundation for support through a Humboldt Research Fellowship.
[1] P. Romanczuk, M. Bär, W. Ebeling, B. Lindner, and L. Schimansky-Geier, Eur. Phys. J. Spec. Top. 202, 1 (2012).

[2] J. Elgeti, R. G. Winkler, and G. Gompper, Rep. Prog. Phys. 78, 056601 (2015).

[3] C. Bechinger, R. Di Leonardo, H. Löwen, C. Reichhardt, G. Volpe, and G. Volpe, Rev. Mod. Phys. 88, 045006 (2016).

[4] A. Zöttl and H. Stark, J. Phys. Condens. Matter 28, 253001 (2016).

[5] T. Vicsek, A. Czirok, E. Ben-Jacob, I. Cohen, and O. Shochet, Phys. Rev. Lett. 75, 1226 (1995).

[6] Y. Hong, N. M. K. Blackman, N. D. Kopp, A. Sen, and D. Velegol, Phys. Rev. Lett. 99, 178103 (2007).

[7] O. Pohl and H. Stark, Phys. Rev. Lett. 112, 238303 (2014).

[8] S. Saha, R. Golestanian, and S. Ramaswamy, Phys. Rev. E 89, 062316 (2014).
[9] B. Liebchen, D. Marenduzzo, I. Pagonabarraga, and M. E. Cates, Phys. Rev. Lett. 115, 258301 (2015).

[10] B. Liebchen, D. Marenduzzo, and M. E. Cates, Phys. Rev. Lett. 118, 268001 (2017).

[11] C. Jin, C. Krüger, and C. C. Maass, Proc. Natl. Acad. Sci. U. S. A. 114, 5089 (2017).

[12] K. Drescher, R. E. Goldstein, and I. Tuval, Proc. Natl. Acad. Sci. USA 107, 11171 (2010).

[13] C. Lozano, B. ten Hagen, H. Löwen, and C. Bechinger, Nat. Commun. 7, 12828 (2016).

[14] J. Palacci, S. Sacanna, A. Abramian, J. Barral, K. Hanson, A. Y. Grosberg, D. J. Pine, and P. M. Chaikin, Sci. Adv. 1, e1400214 (2015).

[15] A. J. T. M. Mathijssen, T. N. Shendruk, J. M. Yeomans, and A. Doostmohammadi, Phys. Rev. Lett. 116, 028104 (2016). 
[16] P. K. Ghosh, Y. Li, F. Marchesoni, and F. Nori, Phys. Rev. E 92, 012114 (2015).

[17] M. P. Magiera and L. Brendel, Phys. Rev. E 92, 012304 (2015).

[18] J. Stenhammar, R. Wittkowski, D. Marenduzzo, and M. E. Cates, Sci. Adv. 2, e1501850 (2016).

[19] A. Geiseler, P. Hänggi, F. Marchesoni, C. Mulhern, and S. Savel'ev, Phys. Rev. E 94, 012613 (2016).

[20] A. Geiseler, P. Hänggi, and F. Marchesoni, Entropy 19, 97 (2017).

[21] A. Geiseler, P. Hänggi, and F. Marchesoni, Sci. Rep. 7, 41884 (2017).

[22] A. Sharma and J. Brader, Phys. Rev. E 96, 032604 (2017).

[23] E. Barry and Z. Dogic, Proc. Natl. Acad. Sci. USA 107, 10348 (2010).

[24] Y. Yang, E. Barry, Z. Dogic, and M. F. Hagan, Soft Matter 8, 707 (2012).

[25] J. N. Israelachvili, S. Marcelja, and R. G. Horn, Q. Rev. Biophys. 13, 121 (1980).

[26] A. Šarić and A. Cacciuto, Phys. Rev. Lett. 108, 118101 (2012).

[27] M. Spellings, M. Engel, D. Klotsa, S. Sabrina, A. M. Drews, N. H. P. Nguyen, K. J. M. Bishop, and S. C. Glotzer, Proc. Natl. Acad. Sci. USA 112, E4642 (2015).

[28] M. E. Cates and J. Tailleur, Annu. Rev. Condens. Matter Phys. 6, 219 (2015).

[29] A. Kaiser, H. H. Wensink, and H. Löwen, Phys. Rev. Lett. 108, 268307 (2012).

[30] A. Kaiser, K. Popowa, H. H. Wensink, and H. Löwen, Phys. Rev. E 88, 022311 (2013).

[31] A. Kaiser, A. Peshkov, A. Sokolov, B. ten Hagen, H. Löwen, and I. S. Aranson, Phys. Rev. Lett. 112, 158101 (2014).

[32] C. Peng, T. Turiv, Y. Guo, Q.-H. Wei, and O. D. Lavrentovich, Science 354, 882 (2016).

[33] P. Guillamat, J. Ignés-Mullol, and F. Sagués, Nat. Commun. 8, 564 (2017).

[34] M. L. Blow, M. Aqil, B. Liebchen, and D. Marenduzzo, Soft Matter 13, 6137 (2017).

[35] T. Kirchhoff, H. Löwen, and R. Klein, Phys. Rev. E 53, 5011 (1996).

[36] H. H. Wensink and H. Löwen, Phys. Rev. E 78, 031409 (2008).

[37] H. H. Wensink, J. Dunkel, S. Heidenreich, K. Drescher, R. E. Goldstein, H. Löwen, and J. M. Yeomans, Proc. Natl. Acad. Sci. USA 109, 14308 (2012).

[38] H. H. Wensink and H. Löwen, J. Phys. Condens. Matter 24, 464130 (2012).
[39] F. C. Keber, E. Loiseau, T. Sanchez, S. J. DeCamp, L. Giomi, M. J. Bowick, M. C. Marchetti, Z. Dogic, and A. R. Bausch, Science 345, 1135 (2014).

[40] R. Sknepnek and S. Henkes, Phys. Rev. E 91, 022306 (2015).

[41] R. Großmann, F. Peruani, and M. Bär, Eur. Phys. J. Spec. Top. 224, 1377 (2015).

[42] W. Li, Sci. Rep. 5, 13603 (2015).

[43] Y. Fily, A. Baskaran, and M. F. Hagan, arXiv:1601.00324 (2016).

[44] L. M. C. Janssen, A. Kaiser, and H. Löwen, Sci. Rep. 7, 5667 (2017).

[45] S. Henkes, M. C. Marchetti, and R. Sknepnek, arXiv:1705.05166 (2017).

[46] S. Shankar, M. J. Bowick, and M. C. Marchetti, Phys. Rev. X 7, 031039 (2017).

[47] R. van Roij, P. Bolhuis, B. Mulder, and D. Frenkel, Phys. Rev. E 52, R1277(R) (1995).

[48] T. Schilling and D. Frenkel, J. Phys. Condens. Matter 16, S2029 (2004).

[49] I. Buttinoni, G. Volpe, F. Kümmel, G. Volpe, I. Buttinoni, G. Volpe, F. Kümmel, G. Volpe, and C. Bechinger, J. Phys. Condens. Matter 24, 284129 (2012).

[50] J. Palacci, S. Sacanna, A. P. Steinberg, D. J. Pine, and P. M. Chaikin, Science 339, 936 (2013).

[51] B. Nitzsche, V. Bormuth, C. Bräuer, J. Howard, L. Ionov, J. Kerssemakers, T. Korten, C. Leduc, F. Ruhnow, and S. Diez, in Microtubules, in vitro, edited by L. Wilson and J. J. Correia, Methods in Cell Biology Vol. 95 (Academic Press, San Diego, CA, 2010), pp. 247-271.

[52] G. Briand and O. Dauchot, Phys. Rev. Lett. 117, 098004 (2016).

[53] J. Rosko, V. A. Martinez, W. C. K. Poon, and T. Pilizota, Proc. Natl. Acad. Sci. USA 114, E7969 (2017).

[54] M. M. Tirado, C. L. Martínez, and J. G. de la Torre, J. Chem. Phys. 81, 2047 (1984).

[55] H. Bartsch, M. Bier, and S. Dietrich, J. Phys. Condens. Matter 29, 464002 (2017).

[56] R. Niu, D. Botin, J. Weber, A. Reinmüller, and T. Palberg, Langmuir 33, 3450 (2017).

[57] G. Junot, G. Briand, R. Ledesma-Alonso, and O. Dauchot (unpublished).

[58] R. Wittmann and J. M. Brader, Europhys. Lett. 114, 68004 (2016).

[59] M. Rex, H. H. Wensink, and H. Löwen, Phys. Rev. E 76, 021403 (2007).

[60] A. M. Menzel, A. Saha, C. Hoell, and H. Löwen, J. Chem. Phys. 144, 024115 (2016). 